\title{
Role of Transperineal Ultrasound (TPUS) in Children with Ambiguous Genitalia
}

\author{
Krithika Rangarajan ${ }^{1}$ Manisha Jana ${ }^{1}$ Nagesh Wadgera ${ }^{1}$ Arun Kumar Gupta ${ }^{1}$ Minu Bajpai ${ }^{2}$ \\ Devasenathipathy Kandasamy ${ }^{1}$
}
${ }^{1}$ Department of Radiology, All India Institute of Medical Sciences, New Delhi, India
2Department of Paediatric Surgery, All India Institute of Medical Sciences, New Delhi, India

\begin{abstract}
Address for correspondence Manisha Jana, MD, DNB, FRCR, Department of Radiology, All India Institute of Medical Sciences, Room 81C, New Delhi, India (e-mail: manishajana@gmail.com).
\end{abstract}

Indian J Radiol Imaging 2021;31:49-56.

\begin{abstract}
Keywords

- ambiguous genitalia

- disorders of sex development

- genitogram

- müllerian structures

- perineum

- transperineal ultrasonography
\end{abstract}

Objectives Accurate delineation of anatomy in children with ambiguous genitalia early in life is important. This commonly involves conventional fluoroscopic genitogram (traumatic to the child) and magnetic resonance imaging (MRI) examination (involves sedation). In this study, our objectives were twofold: (1) to describe the findings on transperineal ultrasound (TPUS) in normal children and (2) to describe the findings on TPUS in children with ambiguous genitalia and correlate them with conventional genitogram.

Materials and Methods TPUS was prospectively performed in 10 children without genital ambiguity ( 5 girls and 5 boys). Subsequently, 15 consecutive children having disorders of sex differentiation (DSDs) with genital ambiguity underwent TPUS. The presence or absence of müllerian structures was documented. Of these patients, 14 also underwent conventional genitogram as a part of routine evaluation. The gold standard was established either by comparison with surgical findings (in patients who underwent surgery) or by comparison with a combination of findings on genitogram and transabdominal ultrasound in patients who did not undergo surgery.

Results In all normal children, lower urogenital tracts could be clearly delineated on TPUS. Out of the 15 children with ambiguous genitalia, TPUS could establish the presence/absence of müllerian structures in 14. This was concordant with findings on conventional genitogram/surgery. In one patient, müllerian structure was missed on TPUS but demonstrated on genitogram. In two children, TPUS showed the müllerian structure, which was not seen on genitogram. When both the controls and the cases were combined, TPUS had an accuracy of $95 \%$ and specificity of $100 \%$ in the detection of müllerian structures.

Conclusion TPUS is feasible and accurate in demonstration of lower urogenital tract anatomy in children with DSDs having ambiguous genitalia. It can be performed without sedation, and is suitable for use as a screening modality in children with ambiguous genitalia. published online April 17, 2021
DOI https://doi.org/

$10.1055 / \mathrm{s}-0041-1729123$

ISSN 0971-3026

\footnotetext{
(c) 2021. Indian Radiological Association

This is an open access article published by Thieme under the terms of the Creative Commons Attribution-NonDerivative-NonCommercial-License, permitting copying and reproduction so long as the original work is given appropriate credit. Contents may not be used for commercial purposes, or adapted, remixed, transformed or built upon. (https://creativecommons.org/licenses/by-nc-nd/4.0/).

Thieme Medical and Scientific Publishers Private Ltd. A-12, Second Floor, Sector -2, NOIDA -201301, India
} 


\section{Introduction}

Ambiguous genitalia in a child are among the most distressing conditions for a parent. It has profound social and psychological consequences. Accurate identification of anatomy is imperative to appropriate gender assignment. The earlier the gender is assigned, the easier the psychological rehabilitation of the child. Management of these children requires a multidisciplinary team including a geneticist, pediatric surgeon, and a pediatric radiologist.

The pediatric radiologist has an important role to play in the accurate identification of anatomy of the internal genital tract of the child. While transabdominal ultrasound is often the first imaging done to identify the gonads (both in male and female children), magnetic resonance imaging (MRI) is often used as a problem-solving tool for evaluation of gonads and internal genital tracts. Nevertheless, conventional genitogram has its own role as the first-line imaging for delineation of the internal genital tracts.

Conventional genitogram is a much widely used technique for evaluation of lower urogenital tract. On the contrary, transperineal ultrasound (TPUS) is not commonly used in evaluation of lower genital tract anatomy in children with disorders of sex differentiation (DSDs) having ambiguous genitalia. TPUS is not a new imaging technique; rather, it has been in practice for the last few decades, both in children and in adults. In children, utility of TPUS is manifold: anorectal malformations, müllerian anomalies, and DSDs; and lower urethral pathologies in male children. ${ }^{1-8}$ The technique is also gaining popularity in pelvic floor studies and incontinence imaging. ${ }^{9-11}$ We undertook this study to describe the findings on TPUS in normal children and subsequently to perform TPUS in children with ambiguous genitalia and compare these findings with conventional genitogram.

\section{Materials and Methods}

\section{Study Design}

This was a prospective study performed with ethical clearance from the institutional review board. Informed consent was obtained from the parents of all the children.

Study period: 6 months (July 2016 to December 2016).

\section{Inclusion Criteria}

The inclusion criteria for the study were:

- Cases: All children scheduled for genitogram during the study period.

- Normal/controls: All children scheduled for a micturating cystourethrogram during the first month of the study period.

\section{Exclusion Criteria}

Children were excluded for the following reason: parental refusal to consent.

\section{Method}

For the first 1 month, only the controls were recruited. TPUS was performed in all of them. For the next 5 months, only the cases were recruited. This was done to standardize the TPUS technique and optimize observer training in interpretation of TPUS. All of the cases underwent genitogram and TPUS on the same day.

\section{Genitography Technique}

Genitography is routinely performed at our institution in children with ambiguous genitalia.

Under proper aseptic precautions, a careful observation of the number and location of the perineal openings were performed. In cases of male child with hypospadias, female child with clitoromegaly, or common urogenital opening, contrast study was performed from the anterior opening (urethra/common channel). The child was positioned in a lateral/steep oblique position. A thin intravenous cannula or hub of an infant feeding tube of smallest caliber ( $5 \mathrm{Fr}$ ) was placed; iodinated contrast was injected and the anatomy was studied under fluoroscopic guidance. It was emphasized that while injecting contrast, the urethra was properly in profile, without any kink/foreshortening. In all cases, fluoroscopic cine-loop images and videos were saved rather than direct exposure. Images were acquired in true lateral position, to avoid any overlap. Another additional frontal image was acquired whenever a müllerian structure was visualized on lateral profile, in order to assess the number and morphology and to evaluate whether it is in the midline. The presence or absence of müllerian structures, degree of development of the müllerian structure (whether fully formed or a small remnant), and length of the common channel were noted.

In the case of cloaca, the hub of a syringe was used for contrast study to avoid any contrast leakage from the wide external opening. For neonates, a syringe of $10-\mathrm{mL}$ capacity was used, and for older infants/children, 20-mL syringe hub was used.

\section{TPUS Technique}

TPUS was performed with a high-frequency (7-12 MHz) linear array transducer. With a generous use of ultrasound jelly, images were acquired in the sagittal and axial plane. On the sagittal plane, the following parameters were noted: the presence or absence of müllerian structure, the presence and length of the common channel, and the distance from the urinary bladder (UB) neck to the junction of the müllerian structure with the urethra (measured in $\mathrm{mm}$ ). The presence or absence of uterus and its appearance (hypoplastic/well-formed) was also noted.

\section{Image Analysis}

Both TPUS and genitogram were analyzed for the following:

- Presence/absence of müllerian structure.

- Presence of a fully formed uterus.

- Visualization and length of the common channel.

The TPUS reader was blinded to results of other investigations and findings of genitogram, and vice versa.

\section{Establishment of Gold Standard}

The gold standard was established by comparison with surgical/laparoscopic findings in five patients who 
underwent surgery. In the rest, a combination of the findings on genitogram and transabdominal ultrasound was considered the gold standard.

\section{Results}

There were 10 control children ( 5 males and 5 females) without genital ambiguity. The age group of both cases and controls ranged between 2 days and 2 years (mean ages were 5 months in cases and 8 months in control group). The older children had cloacal malformation and congenital adrenal hyperplasia (CAH).

In the control group, on TPUS, three tracts could be seen in all female children corresponding to the urethra, vagina, and the anal canal ( $\boldsymbol{- \text { Fig. }} \mathbf{1}$ ). TPUS of all male children showed only two tracts: the urethra and the anal canal (-Fig. 2). Each tract was seen as a hypoechoic wall with a strip of fluid (anechoic) and echogenic mucosa. Intraluminal foci of air were seen as echogenic areas.

Of the 15 children with ambiguous genitalia, 14 underwent both TPUS and genitogram. One child was only 2 days old and therefore only underwent TPUS, as the parents did not give consent for performing a genitogram.

Out of 15 children with ambiguous genitalia, 6 were being reared up as male and referred for evaluation of hypospadias with or without undescended/absent testis, 4 were being reared up as female and had enlarged clitoris with suspicion of CAH, 3 had a common cloaca, and 2 patients had a common urogenital opening with varying degrees of labial fusion ( - Table 1). We divided the patients (according to the clinical findings) into three subsets:

- Group 1: hypospadias.

- Group 2: CAH.

- Group 3: common urogenital sinus (UGS) or common cloaca. This group was further analyzed in two separate sets:

- Common UGS.

- Common cloaca.

In group 1 (hypospadias), all six children showed an external male appearance, with undescended impalpable testes. TPUS did not show müllerian structure in any of them ( - Fig. 3). Genitogram revealed male-type urethra and no müllerian structure.

In group 2 (CAH), all four children had variable degree of clitoromegaly. On TPUS, three of these four children were

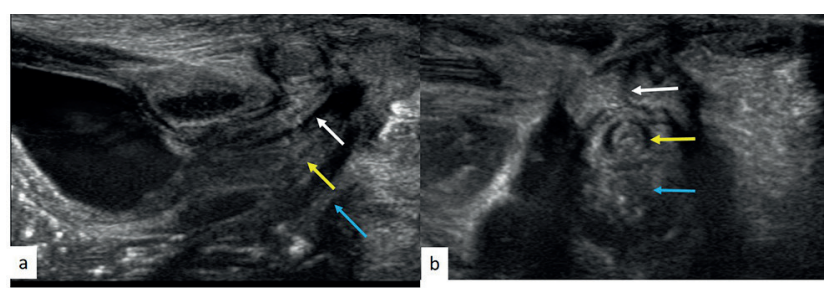

Fig. 1 Normal female anatomy on TPUS. TPUS in a female child without genital ambiguity clearly shows three tracts corresponding to the urethra (white arrow), vagina (yellow arrow), and anal canal (blue arrow) on (a) sagittal and (b) axial images.

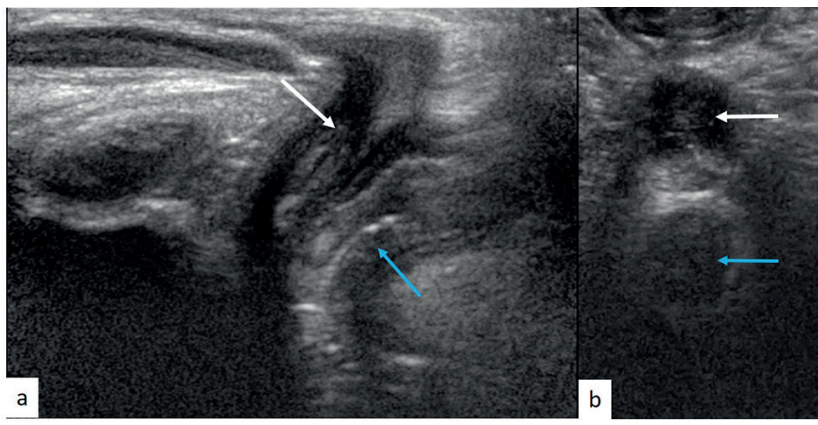

Fig. 2 Normal male anatomy on TPUS. TPUS in a male child without genital ambiguity shows only two tracts corresponding to urethra (white arrow) and anal canal (blue arrow) on (a) sagittal and (b) axial images.

Table 1 Final diagnosis of the cases

\begin{tabular}{|l|l|}
\hline Clinical diagnosis & Number of patients \\
\hline Hypospadias (group 1) & $6(40 \%)$ \\
\hline CAH (group 2) & $4(26.7 \%)$ \\
\hline Common cloaca (group 3) & $3(20 \%)$ \\
\hline Variation of labial fusion (group 3) & $2(13.3 \%)$ \\
\hline Lower vaginal atresia with UGS & 1 \\
\hline UGS only & 1 \\
\hline Total & $15(100 \%)$ \\
\hline
\end{tabular}

Abbreviations: CAH, congenital adrenal hyperplasia; UGS, urogenital sinus.

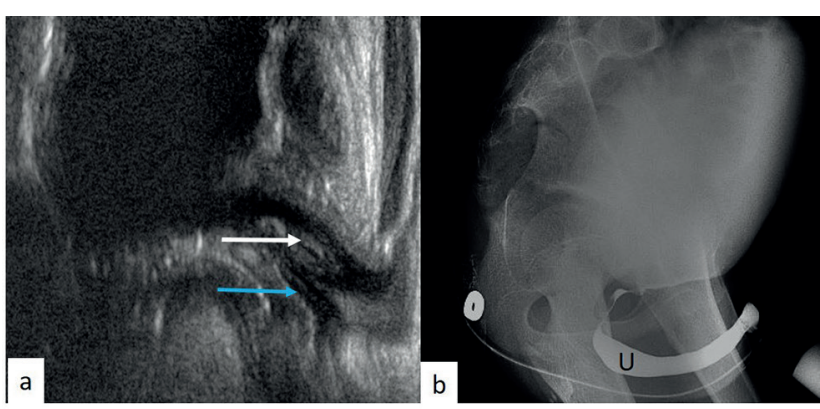

Fig. 3 A patient with hypospadias. (a) TPUS sagittal section (rotated 90 degrees for the sake of similarity in comparison with genitogram) shows only two tracts (urethra: white arrow; rectum: blue arrow) without müllerian structures. (b) Short male-type urethra $(U)$ seen on genitogram without any müllerian structure.

seen to have three separate tracts corresponding to the urethra, vagina, and anal canal. The urethra and vaginal tract merged caudally; the junction was variable in different patients. TPUS was able to demonstrate the presence of uterus in all. Well-developed uterus and ovaries were also seen on transabdominal ultrasound in all.

However, in one patient, diagnostic quality images could not be obtained on TPUS. The images were interpreted as showing only two tracts: the urethra and anal canal. Müllerian structures/remnants were missed on TPUS in this patient. 
All four of them showed well-developed müllerian structures with a cervical impression on genitogram. These children were proven to be CAH based on laboratory results.

In group 3 (common UGS or common cloaca), all the five patients presented with varying degrees of labial fusion or truly ambiguous external genital appearance. Of these children, two had common urogenital opening and three had common cloaca.

Of the two children with a common urogenital opening, both showed the presence of three tracts on TPUS. One revealed a blind-ending vagina without any uterus ( - Fig. 4), and the other showed presence of uterus. Abdominal ultrasound confirmed the presence of uterus in this second patient. Genitogram was performed in only one child of this group, and it revealed presence of a blind-ending müllerian structure, concordant with the TPUS finding. The findings of TPUS were found concordant on surgery.

All three children with a common cloaca were operated early in life with a diversion colostomy and presented for evaluation of the urogenital tract. Of these, in one child an ovary was seen on transabdominal scan and a testicle was seen in the left scrotum on ultrasound ( - Fig. 5). TPUS showed three tracts, and genitogram also revealed a well-developed uterus and vagina. This child was diagnosed as ovotesticular DSD. In two other children, fluid-filled structure was seen on TPUS in between the urethra and anal canal, but no müllerian structure was seen on genitogram. One of them was found to be an obstructed müllerian structure on surgery and another was found to be vaginal atresia ( - Fig. 6).

The delineation of lower genital tract anatomy was separately evaluated for TPUS and genitogram. In delineating anatomy, the parameter assessed was presence or absence of müllerian structure. The findings were compared with the gold standard. The findings are summarized in - Table 2 .

The sensitivity of TPUS and genitogram (-Table 2) are 88.88 and $75 \%$, respectively, with the specificity of $100 \%$ in both. Genitogram had lower sensitivity as it could not detect müllerian structures in two cases (one in obstructed müllerian structure and another in vaginal atresia).
The spectrum of appearance of müllerian structure is described in - Table 3 . TPUS could demonstrate müllerian structures in eight out of nine patients, whereas genitogram could do so in six out of eight patients (genitogram not performed in one patient of this group).

\section{Length of Common Channel}

The analysis of length of the common channel was performed in groups 2 and 3. On genitogram, the length of the common channel was measured from the junction (of the müllerian structure and the urethra) to the tip of the urethra. This measurement was not technically possible on TPUS due to nonvisualization of the entire extent in a single image/plane. On TPUS, the distance from the UB neck to the junction of the müllerian structure with urethra was measured ( - Fig. 7).

\section{Discussion}

Children whose external genitalia do not fit the anatomical configuration of male or female external genitals are said to have ambiguous genitalia. To counter the use of terms that were considered potentially pejorative (such as intersex and sex reversal), the term "disorders of sex differentiation" (DSDs) has been proposed in the International Consensus Conference on Intersex. ${ }^{12,13}$

According to the recent terminology, DSDs can be broadly summed up into three categories: 46, XY DSD (earlier known as male pseudohermaphroditism); 46, XX DSD (earlier known as female pseudohermaphroditism); and sex chromosome DSD (such as $45, \mathrm{X}$ or $47, \mathrm{XXY}$ ). ${ }^{14}$

However, it has to be remembered that not all children with DSD present with an ambiguous genitalia. In some cases, the external genitalia may be that of male/female; yet the chromosomal sex may not be conforming to the external appearance. ${ }^{14,15}$ An example of such situation is complete androgen insensitivity syndrome (AIS), wherein the patient is phenotypically a female (no genital ambiguity) and bears a chromosomal sex of 46 , XY. The presence of an ambiguous genitalia points toward DSD, but not all DSDs have ambiguous genitalia.

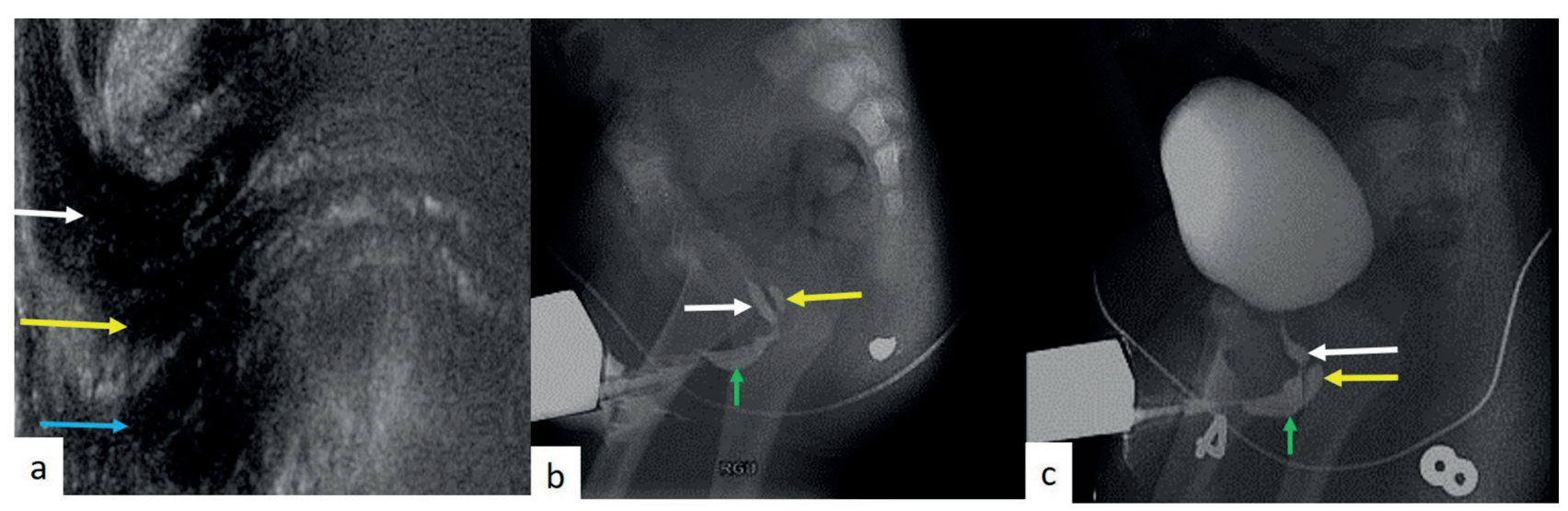

Fig. 4 (a) Common urogenital sinus. TPUS sagittal section (rotated 90 degrees for the sake of similarity in comparison with genitogram) shows the presence of a tract (with upward tapering) representing a blind müllerian structure (yellow arrow). No uterus was seen on TAS of the same patient. (b, c) Genitogram of the same child showed the presence of a müllerian structure without a cervical impression (yellow arrow). Urethra is shown with white arrow, anal canal with blue arrow, and common channel with green arrow. 


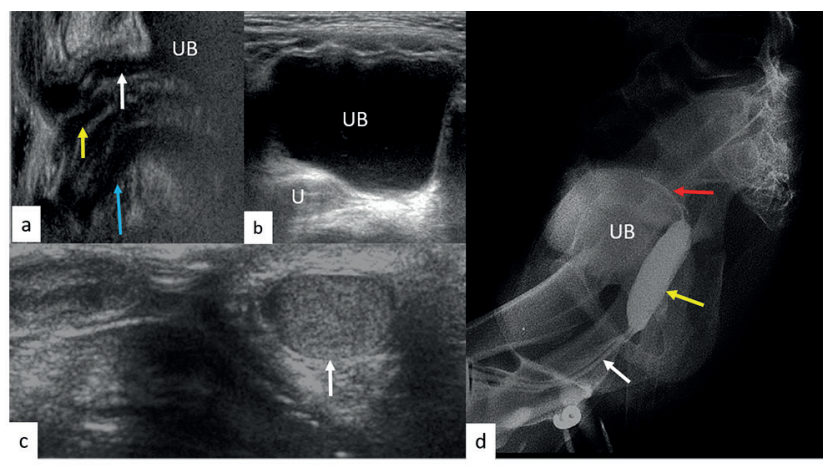

Fig. 5 Ovotesticular DSD. (a) TPUS sagittal section (rotated 90 degrees for the sake of similarity in comparison with genitogram) shows presence of three tracts: urethra (white arrow), vagina (yellow arrow), and anal canal (blue arrow). (b) Uterus (U) is seen on axial transabdominal ultrasound. (c) Testicle (arrow) is also seen in the left scrotal sac. (d) Genitogram shows catheter in the urethra (white arrow), contrast distending the vagina (yellow arrow), and a contrast streak in the uterine cavity (red arrow).
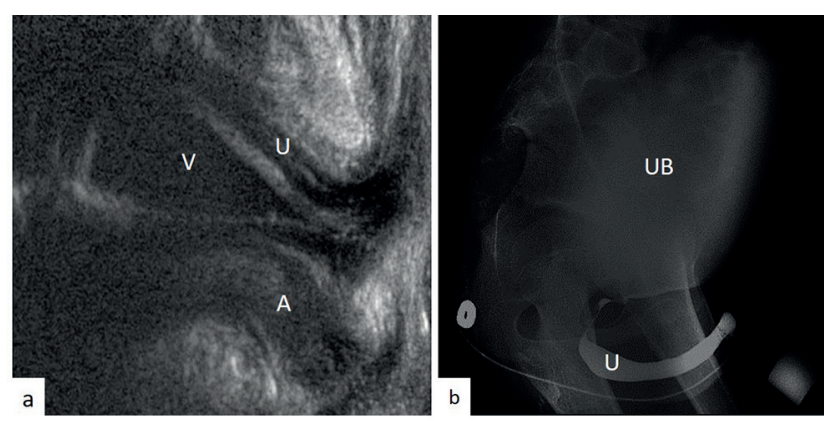

Fig. 6 (A and B) Distal vaginal atresia. (A) TPUS sagittal section (rotated 90 degrees for the sake of similarity in comparison with genitogram) shows the presence of a round-topped fluid filled structure $(\mathrm{V})$ in between the urethra $(\mathrm{U})$ and anal canal (A). (B) Genitogram reveals a male type urethra $(U)$; but does not show the presence of any müllerian structure. (B) On surgery, distal vaginal atresia was seen.

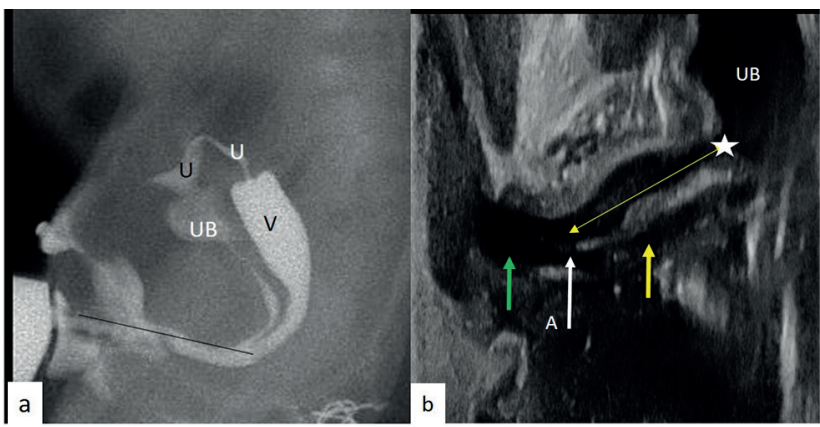

Fig. 7 Ambiguous genitalia in congenital adrenal hyperplasia. Measurement of common channel length/its surrogate. (a) Measurement on genitogram. The length is shown with black line. It is measured from the junction (of the mülerian structure with the urethra) to the tip of the urethra. $U$, endometrial cavity; $V$, vagina. (b) Measurement on sagittal TPUS image rotated 90 degrees for the sake of similarity in comparison with genitogram. Distance (double-sided arrow) measured from the bladder neck (asterisk) till the point of junction of both the channels (white arrow). Common channel is shown with a green arrow and vagina with yellow arrow. A, anal canal; UB, urinary bladder.

Common cause of 46, XX DSD who have ambiguous genitalia is CAH (incomplete virilization type). $46, \mathrm{XY}$ DSDs, which commonly have ambiguous genitalia, include incomplete AIS and $5 \alpha$-reductase deficiency. Ovotesticular DSD is a rare disorder presenting with ambiguous genitalia and variable combination of abnormal gonadal tissue (ovary/testis/ovotestis). ${ }^{16}$ The chromosomal sex in ovotesticular DSD is also variable.

Genital ambiguity is most often recognized in the neonatal period. There may be frank genital ambiguity in the form of a common cloaca; the children present with undermasculinized male genitalia (micropenis, hypospadias, undescended testis) or virilized female genitalia (enlarged clitoris or with varying degrees of labial fusion or a mass in the

Table 2 Visualization of müllerian structures on genitogram and TPUS

\begin{tabular}{|c|c|c|c|c|}
\hline & Clinical presentation & $\begin{array}{l}\text { Visualization of müllerian } \\
\text { structures on TPUS ( } n=25 \text {, } \\
\text { including } 10 \text { controls) }\end{array}$ & $\begin{array}{l}\text { Visualization of } \\
\text { müllerian structures on } \\
\text { genitogram }(n=14)\end{array}$ & Gold standard \\
\hline Group 1 & Hypospadias $(n=6)$ & 0 & 0 & No müllerian structures \\
\hline Group 2 & $\mathrm{CAH}(n=4)$ & $3(75 \%)$ & $4(100 \%)$ & $\begin{array}{l}\text { All had well-developed müllerian } \\
\text { structures }\end{array}$ \\
\hline Group 3 & $\begin{array}{l}\text { Common cloaca/ } \\
\text { common urogenital } \\
\text { opening }(n=5)\end{array}$ & $5(100 \%)$ & $2(\text { out of } 4)^{a}(50 \%)$ & $\begin{array}{l}\text { All had some müllerian struc- } \\
\text { tures of varying degrees of } \\
\text { development }\end{array}$ \\
\hline $\begin{array}{l}\text { Control } \\
\text { (male) }\end{array}$ & & 0 & & \\
\hline $\begin{array}{l}\text { Control } \\
\text { (female) }\end{array}$ & & $5(100 \%)$ & & \\
\hline Total & & 8/25 (TP-8, TN-16, FP-0, FN-1) & $\begin{array}{l}\text { 6/14 (TP-6, TN-6, FP-0, } \\
\text { FN-2) }\end{array}$ & \\
\hline
\end{tabular}

Abbreviations: CAH, congenital adrenal hyperplasia; TPUS, transperineal ultrasound; FN, false negative; FP, false positive; TN, true negative; TP, true positive; NPV, negative predictive value; PPV, positive predictive value.

Note: Müllerian structures on TPUS: sensitivity $=88.88 \%$, specificity $=100 \%, P P V=100 \%, N P V=94.11 \%$, accuracy $=95 \%$. Müllerian structures on genitogram: sensitivity $=75 \%$, specificity $=100 \%, P P V=100 \%, N P V=75 \%$, accuracy $=85.71 \%$.

${ }^{a}$ Genitogram was not performed in one. 
Table 3 Spectrum of müllerian structures on imaging ( $n=9$, excluding hypospadias group)

\begin{tabular}{|l|l|l|l|}
\hline Appearance & No. & Detected on TPUS & Detected on genitogram \\
\hline Well-formed uterus and vagina (4 CAH, 1 cloaca) & 5 & 4 & 5 \\
\hline Poorly formed vagina, no uterus & 3 & 3 & $1^{\text {a }}$ \\
\hline Vaginal atresia, no uterus & 1 & 1 & 0 \\
\hline
\end{tabular}

Abbreviations: CAH, congenital adrenal hyperplasia; TPUS, transperineal ultrasound.

${ }^{a}$ Genitogram was not performed in 1 .

inguinal or labial region)..$^{13}$ Clinical evaluation should take into account the presence or micropenis versus clitoromegaly, palpable versus impalpable gonads, number of orifices in the perineum, and location of urethral orifices. ${ }^{16}$

Radiological investigation comes next, and common investigations advocated in clinical practice are pelvic US for gonads, genitogram for lower urogenital tract anatomy, and MRI as an additional/problem-solving modality for both internal genital organs and gonads..$^{14,17-19}$ MRI has been found to be very useful in demonstration of gonads, differentiation of phallus from the enlarged clitoris, and the presence of some complex anomalies. ${ }^{20-22}$ However, its cost and requirement of sedation/anesthesia remain main points of consideration, especially when used in infants/small children.

Identification of internal genital tract and gonads is an essential component of radiological evaluation. Radiological findings are considered together with karyotyping and hormonal assessment to reach a final diagnosis. ${ }^{23}$ Genitogram involves placement of a small catheter in the urogenital opening and injection of an iodinated contrast agent. In combination with pelvic ultrasonography, it is very useful to delineate urogenital anatomy. ${ }^{24}$ Being a fluoroscopic procedure, it involves irradiation to the gonads of these young children, in addition to being traumatic due to placement of the catheter. Also, a study by Vanderbrink et al on children with CAH reported that genitogram findings were seen to be inaccurate at demonstrating urogenital anatomy in up to $25 \%$ of cases. ${ }^{25}$

In our institute, genitogram is routinely performed for children with ambiguous genitalia to help identify müllerian structures and to accurately delineate lower urogenital tract anatomy. In the case of the presence of a common channel, genitogram also helps measure the length of the common channel.

While both genitogram and MRI have their own imaging advantages, there is also a need for some imaging technique that can be quickly performed on a small child without sedation or without the risk of catheterization and ionizing radiation. TPUS perfectly fits in this situation as a first-line screening imaging tool in ambiguous genitalia. TPUS has been tried in this scenario by some investigators earlier, but no specific imaging guidelines regarding its utility in DSD are widely available. ${ }^{26-28}$

Our study demonstrated the ability of the technique to elucidate the anatomy of pelvic organs. In all the children without genital ambiguity, TPUS clearly demonstrated the tracts of the urethra, anal canal, and vagina in female children. In groups 1 and 2, both TPUS and genitogram were fairly accurate in delineating lower genital tract anatomy.
However, in group 3, TPUS was better than genitogram. This group consisted of complex anomalies such as cloaca, vaginal atresia, and obstructed müllerian structure. Genitogram could not demonstrate the müllerian structures when it did not communicate with the urethra. Across all the three groups, in terms of detection of müllerian structures, TPUS has higher sensitivity than genitogram ( 88.9 vs. $75 \%$ ).

When both the controls and the cases were combined, TPUS had an excellent accuracy of $95 \%$ and specificity of $100 \%$ in the delineation of anatomy and detection of müllerian structures. TPUS also was accurate in assessing the degree of development of the müllerian structures. Moreover, all the children tolerated the noninvasive procedure of TPUS better than the invasive genitogram.

TPUS was inaccurate and missed müllerian structure in one child, where images of diagnostic quality could not be obtained. This was possibly because of the child's body habitus (large amount of labial fat). Also, unlike in genitogram where the length of the common channel (which is of great surgical importance) could be measured directly, this was not possible in TPUS. In an earlier study by Lindert et al, length measurement of the common channel was performed on TPUS in a way similar to usual genitographic measurement, and yielded reasonably good correlation with cystoscopic estimate of common channel length. ${ }^{27}$ However, in our experience, this method did not result in a very accurate estimate of the common channel length. One explanation to this phenomenon may be the fact that they have children of higher age group compared with ours. Similar to our experience, in the case of vaginal atresia, TPUS was shown to be useful in congenital vaginal anomalies such as atresia in earlier studies as well. ${ }^{25,27}$

Our study re-emphasized the utility of TPUS as a quick screening tool along with routine transabdominal ultrasonography in cases of DSD. - Fig. 8 describes a suggested simplified algorithmic approach to a child with genital ambiguity. It can be noted that to reach an etiological diagnosis, a combination of pelvic US and TPUS with karyotyping may be sufficient. This is because the etiological diagnosis mostly depends on the presence and morphology of müllerian structures and visualization of gonads. Both of the information can be provided by a combination of pelvic US and TPUS. Exceptions include streak gonads or undescended testes nonvisualized by US.

As a surrogate for the length of the common channel, the distance of the junction of both the tracts from the UB neck can be measured.

Moreover, TPUS has some distinct advantages over genitogram in terms of an absence of radiation exposure and being 


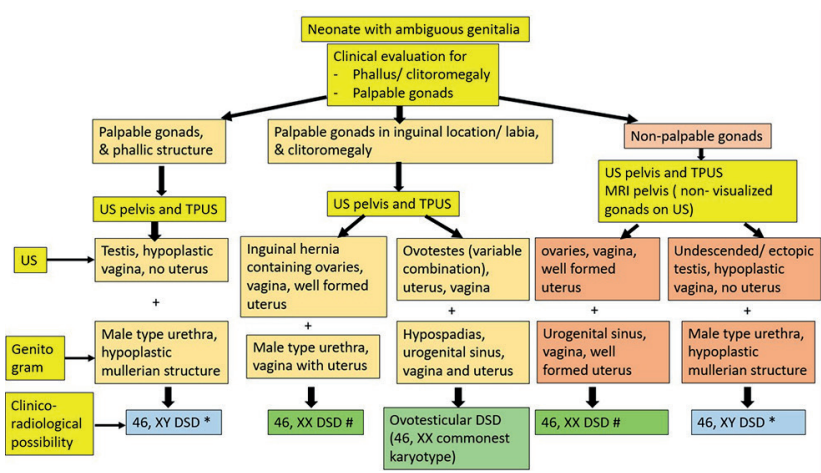

Fig. 8 Suggested clinical and imaging algorithmic approach to a child with genital ambiguity ${ }^{*}$ Common etiologic possibility: partial AIS and $5 \alpha$-reductase deficiency. They can present with either palpable (descended) or nonpalpable (undescended/ectopic) gonads. \#Common etiological possibility: CAH (partial virilization type). When associated with inguinal hernia containing ovaries, the gonads may be palpable. Degree of virilization is also variable.

less traumatic to the child. In comparison to MRI, TPUS is much cheaper and faster and does not require sedation.

Our study had some limitations in that the size of the study was very small (only 15 patients with genital ambiguity). Besides, we did not compare the TPUS images with MRI, which is widely used now for evaluation of such patients. A larger study that compares findings on TPUS with surgical findings as well as MRI would help further investigation of the modality.

To conclude, while genitogram remains the first and most preferred imaging technique for evaluation of lower genital tract anatomy, and MRI is ideal and is often used as problem-solving tool, TPUS can have its own niche indication as a quick screening tool for lower genital tract in children presenting with ambiguous genitalia.

\section{Declaration of Patient Consent}

The authors certify that they have obtained all appropriate patient consent forms. In the form, the patient(s) has/have given his/her/their consent for his/her/their images and other clinical information to be reported in the journal. The patients understand that their names and initials will not be published and due efforts will be made to conceal their identity, but anonymity cannot be guaranteed.

\section{Financial Support and Sponsorship}

Nil.

\section{Conflict of Interest}

The authors declare no conflict of interest.

\section{References}

1 Haber HP. Ultrasonography of imperforate anus in neonate: an approach correlated with current surgical concepts. Ultraschall Med 2009;30(2):189-195

2 Haber HP, Seitz G, Warmann SW, Fuchs J. Transperineal sonography for determination of the type of imperforate anus. AJR Am J Roentgenol 2007;189(6):1525-1529
3 Choi YH, Kim IO, Cheon JE, Kim WS, Yeon KM. Imperforate anus: determination of type using transperineal ultrasonography. Korean J Radiol 2009;10(4):355-360

4 Kim IO, Han TI, Kim WS, Yeon KM. Transperineal ultrasonography in imperforate anus: identification of the internal fistula. J Ultrasound Med 2000;19(3):211-216

5 Alamo L, Meyrat BJ, Meuwly JY, Meuli RA, Gudinchet F. Anorectal malformations: finding the pathway out of the labyrinth. Radiographics 2013;33(2):491-512

6 Hamed ST, Mansour SM. Surface transperineal ultrasound and vaginal abnormalities: applications and strengths. $\mathrm{Br} \mathrm{J}$ Radiol 2017;90(1080):20170326

7 Paltiel HJ, Phelps A. US of the pediatric female pelvis. Radiology 2014;270(3):644-657

8 Laufer RM. Structural abnormalities of the female reproductive tract. In: Emans SJ, Laufer MR, eds. Goldstein's Pediatric and Adolescent Gynecology. 7th ed. Philadelphia, PA: Lippincott Williams \& Wilkins; 2019 99-144

9 Amin MA, Eltomey MA, El-Dorf AA. Role of transperineal ultrasound measurements in women with prolonged second stage of labor as predictors of the mode of delivery. Egypt J Radiol Nucl Med 2014;45:1295-1299

10 Roche B, Zufferey G, Robert-Yap J. Transperineal ultrasonography. In: Santoro GA, Wieczorek AP, Bartram CI, eds. Pelvic Floor Disorders. Milano: Springer; 2010 297-303

11 Valsky DV, Yagel S. Three-dimensional transperineal ultrasonography of the pelvic floor: improving visualization for new clinical applications and better functional assessment. J Ultrasound Med 2007;26(10):1373-1387

12 Lee PA, Houk CP, Ahmed SF, Hughes IA. International Consensus Conference on Intersex organized by the Lawson Wilkins Pediatric Endocrine Society and the European Society for Paediatric Endocrinology. Consensus statement on management of intersex disorders. Pediatrics 2006;118(2):e488-e500

13 Hughes IA, Houk C, Ahmed SF, Lee PA. LWPES Consensus Group. ESPE Consensus Group. Consensus statement on management of intersex disorders. Arch Dis Child 2006;91(7):554-563

14 Chavhan GB, Parra DA, Oudjhane K. Miller SF, Babyn PS, Pippi Salle FL. Imaging of ambiguous genitalia: classification and diagnostic approach. Radiographics 2008;28(7):1891-1904

15 Moshiri M, Chapman T, Fechner PY, et al. Evaluation and management of disorders of sex development: multidisciplinary approach to a complex diagnosis. Radiographics 2012;32(6):1599-1618

16 Indyk JA. Disorders/differences of sex development (DSDs) for primary care: the approach to the infant with ambiguous genitalia. Transl Pediatr 2017;6(4):323-334

17 Guerra-Junior G, Andrade KC, Barcelos IHK, Maciel-Guerra AT. Imaging techniques in the diagnostic journey of disorders of sex development. Sex Dev 2018;12(1-3):95-99

18 Choi HK, Cho KS, Lee HW, Kim KS. MR imaging of intersexuality. Radiographics 1998;18(1):83-96

19 Al Jurayyan NA, Al-Jurayyan RN, Mohamed SH, Babiker AM, Al Otaibi HM. Radiological imaging of disorders of sex development (DSD) Sudan J Paediatr 2013;13(2):10-16

20 Kim KS, Kim J. Disorders of sex development. Korean J Urol 2012;53(1):1-8

21 Hricak H, Chang YC, Thurnher S. Vagina: evaluation with MR imaging. Part I. Normal anatomy and congenital anomalies. Radiology 1988;169(1):169-174

22 Alaniz VI, Kobernik EK, Dillman J, Quint EH. Utility of ultrasound and magnetic resonance imaging in patients with disorders of sex development who undergo prophylactic gonadectomy. J Pediatr Adolesc Gynecol 2016;29(6):577-581

23 El-Ella SS, Tawfik MA, Abo El-Fotoh WM, Abo Howla AS. Genetic evaluation of children with ambiguous genitalia. Menoufia Med J 2016;29:79-88 
24 al Jurayyan NA, Patel PJ, al Herbish AS, et al. Ambiguous genitalia: comparative role of pelvic ultrasonography and genitography. Ann Trop Paediatr 1995;15(3):203-207

25 Vanderbrink BA, Rink RC, Cain MP, et al. Does preoperative genitography in congenital adrenal hyperplasia cases affect surgical approach to feminizing genitoplasty? J Urol 2010;184(4(Suppl):1793-1798
26 Scanlan KA, Pozniak MA, Fagerholm M, Shapiro S. Value of transperineal sonography in the assessment of vaginal atresia. AJR Am J Roentgenol 1990;154(3):545-548

27 Lindert J, Hiort O, Tüshaus L, Tafazzoli-Lari K, Wünsch L. Perineal ultrasound offers useful information in girls with congenital adrenal hyperplasia. J Pediatr Urol 2016;12(6): 427.e1-427.e6 